Egyptian

Orthodontic Journal

\title{
EXTRACTION TREATMENT AND SOFT TISSUE FACIAL PROFILE
}

\author{
Ahmed R. Afify*
}

ABSTRACT

A harmonious soft-tissue facial profile is an important consideration in orthodontic diagnosis and treatment planning. Extraction of premolar teeth sometimes is necessary to achieve the treatment goals. Facial soft tissue profile adaptation to incisors retraction is still controversial. This study was carried out to evaluate the response of soft tissue facial profile to maxillary and mandibular incisors retraction. Pre and post treatment lateral cephalograms of 30 patients, having bimaxillary dental protrusion, who required extraction of four first premolars, were traced, superimposed, and evaluated. All patients were treated with preadjusted straight wire appliances. All the subjects were females with a mean age of 16 years, 5 months to reduce variables caused by growth and sex differences. The Facial soft tissue profile was evaluated using a series of eight linear and angular measurements, and the effect of orthodontic correction was determined using paired t-tests, then Pearson $r$ correlation analysis was done to calculate the correlation coefficient. The results demonstrated that the extraction of four premolars greatly reduces soft tissue procumbency seen in patients with bimaxillary protrusion that were indicated by the significant post treatment changes for all of the soft tissue profile variables studied. Pearson $r$ correlation analysis indicated that:

- The Nasolabial angle, Lower lip retraction and Upper lip retraction correlates well with upper incisors retraction.

- Lower lip retraction correlates well with lower incisors retraction.

\footnotetext{
* Assistant Professor, Department of Orthodontics, Faculty of Dentistry, Mansoura University, Egypt.
} 
Egyptian

Orthodontic Journal

- Upper lip retraction correlates well with the lower lip retraction.

- Labiomental angle does not show good correlation with upper incisors retraction.

- Lower lip thickness does not correlate with upper incisors retraction.

- Upper lip thickness showed poor correlation with Upper incisors retraction.

\section{INTRODUCTION}

The attainment of an optimum facial soft-tissue profile is one of the principal goals in the planning of treatment of orthodontic problems. The debate concerning the extraction of teeth and its effect on the facial profile began more than 100 years ago.

Bimaxillary protrusion is characterized by protrusive teeth in both jaws and a greater than average degree of lip prominence. Often the sole purpose of extracting premolars in patients with bimaxillary protrusion is to create space to retract the anterior teeth in order to reduce the procumbency of the lips. Many of our pioneers in the orthodontic field have given their opinions concerning the relationship between the soft tissue integumental profile and the underlying skeletal structures. ${ }^{1-8}$

Some researchers ${ }^{9-12}$ have found a high degree of correlation between incisor and upper lip retraction, suggesting a close relationship between the softtissue profile and the position of the maxillary incisor. Other investigators ${ }^{7,13-16}$ have found that proportional change or improvement in the soft-tissue profile does not necessarily accompany extensive changes in the dentition. The variance in soft-tissue veneer overlying the skeletal structures is a factor proposed to account for this lack of correlation between hard and soft tissues.

Riedel $^{6}$ was one of the first investigators who studied the response of the soft-tissue profile to orthodontic treatment. He reported that the relation of the maxillary and mandibular apical bases, the degree of convexity of the skeletal pattern, and the relation of the anterior teeth to their respective apical bases have a marked influence on the soft-tissue profile.

Holdaway ${ }^{17}$ proposed a method of analyzing the soft-tissue profile both as a means of diagnosis and as a method for determining changes in the soft-tissue profile induced by growth and treatment. He proposed the " $\mathrm{H}$ " angle, formed by a line drawn from the point of the soft-tissue chin tangent to the upper lip and 
the N-B line, as a measure of profile acceptability. He believed that the $\mathrm{H}$ angle should be 7 to 9 degrees when the ANB angle is 3 degrees. The angle could be adjusted to compensate for changes in the ANB angle to alter lip fullness.

Lip structure seems to have an influence on lip response to incisor retraction. Oliver ${ }^{18}$ found that patients with thin lips or a high lip strain displayed a significant correlation between incisor retraction and lip retraction, whereas patients with thick lips or low lip strain displayed no such correlation. In addition, Wisth ${ }^{19}$ found that lip response, as a proportion of incisor retraction, decreased as the amount of incisor retraction increased. This seems to indicate that the lips have some inherent support.

Perkins ${ }^{20}$ found that decreases in upper vermilion height were positively correlated with upper lip retraction. However, changes in the upper vermilion height did not significantly correlate with the magnitude of incisor retraction.

The purpose of this study was to investigate the soft-tissue profile changes caused by retraction of the maxillary and mandibular incisors, and identifying and quantifying the contribution of the most significant factors responsible for these changes.

\section{Materials And Methods}

The sample consisted of 30 Angle Class I cases. They were selected from the outpatient Clinic of Orthodontic Department, Faculty of Dentistry, Mansoura University. All the subjects were females and treated by extraction of the four first premolars. First Premolar teeth were extracted for the relief of crowding and reduction of incisors protrusion.

Both the pretreatment and posttreatment lateral cephalograms were taken in centric occlusion with the same cephalometer. Lateral cephalograms were taken with Frankfort horizontal parallel to the floor and with patient's lips in repose to minimize potential sources of lip strain that could influence the softtissue profile. ${ }^{7}$

The selection criteria for those patients were as follows:

1. Angle's Class I with bimaxillary protrusive profile as determined by clinical soft tissue profile assessment.

2. Moderate mandibular plane angle as evidenced by the Frankfort mandibular angle between $23^{\circ}$ to $28^{\circ}$ determined through the pretreatment lateral cephalograms.

3. All patients were Egyptian adult females ranging in age from 14 years, 9 months to 20 years, 4 months with the mean age of 16 years, 5 months, at the start of orthodontic treatment. Only post pubertal patients were selected. 
4. At least $3 \mathrm{~mm}$ lower incisor retraction was necessary to be included in this study.

5. High quality pre- and post treatment lateral cephalograms, each exhibiting good soft tissue definition with lips relaxed, teeth in occlusion, and taken using the same cephalostat, were available for all subjects.

6. At the end of treatment, all patients were considered to be well treated, as evidenced by Class I canine and molar relationships, and had overbites between $10 \%$ and $25 \%$; both dental arches were well aligned, with teeth interdigitated.

All patients had been treated with straight wire preadjusted 0.022 " slot Roth prescription. None of the patients was treated with expansion devices; however, interarch elastics and headgears were used as required.

\section{Cephalometric analysis:}

All cephalograms were taken with patients in a standing position with relaxed lips. The patients were asked to close on the molars and not to stress the lips. The relaxed lip position has been suggested as the best posture for cephalometric softtissue evaluation. ${ }^{21,22}$ All the cephalograms were taken using the same machine to eliminate the need for correction of radiographic magnification. Posttreatment cephalograms were taken within 1 month of appliance removal. The films were carefully traced for each patient on 8 by 10 inch acetate paper. Each pair of radiographs of a patient was traced at the same sitting to minimize tracing errors. All measurements were taken to the nearest $0.5 \mathrm{~mm}$ or 0.5 degree. Assessment of error involved in the radiographic measurement was done through retracing and measuring the films of 10 subjects one month after the initial measurements. Results of the paired $t$-test showed no significant differences between the two sets of measurements, at the $95 \%$ confidence level .

The following skeletal, dental, and soft-tissue landmarks were identified for the study (Fig. 1):

Skeletal: Sella $(S)$ - Center of the contour of sella turica. Nasion $(N)$ - Most anterior point of the frontonasal suture in the midsagittal plane. Dental: Upper incisor point (Uip) - The most anterior point on the crown of the upper incisor. Lower incisor point (Lip) - The most anterior point on the crown of the lower incisor. Soft tissue: Subnasale $(S n)$ - The point of convergence of the nose and the upper lip. Superior sulcus $(\mathrm{Ss})$ - The point of greatest concavity in the midline between the upper lip (Ls) and subnasale (Sn). Labrale superius (Ls) - The most anterior point on the convexity of the upper lip. Labrale inferius ( $\mathrm{Li}$ ) - The most anterior point on the convexity of the lower lip. Sulcus inferius ( $\mathrm{Si}$ — - The point of greatest concavity in the 
midline between the lower lip and soft-tissue chin. Soft-tissue pogonion $\left(\operatorname{Pog}^{\prime}\right)$ - The most anterior point of the soft-tissue chin.

\section{Reference lines: (Fig. 2)}

Since the sella and nasion are much more reproducible landmarks the measurements are made relative to the constructed Frankfort horizontal line at $7^{\circ}$ inferior to the S-N line through sella as the horizontal plane of reference and a vertical line drawn from the "sella" at $90^{\circ}$ to the constructed Frankfort horizontal line as the vertical plane of reference. ${ }^{23,24}$

\section{Measurements:}

Two angular and six linear measurements were selected to examine changes in the soft-tissue profile: (Fig 2) Angular measurements: 1-Nasolabial angle - Formed by intersection of a line originating in Sn,tangent to lower margin of the nose, and a line traced between Sn and Ls. 2-Labiomental angle Formed by intersection of a line traced between $\mathrm{Li}$ and $\mathrm{Si}$ and a line traced between Si and Pog'.

Linear measurements: 1- Upper lip thickness was measured as the distance between the most anterior point on the lip (LS) and the most prominent point on the labial surface of the incisor (IA). 2- Lower lip thickness was measured as the distance between the most anterior point on the lip (LI) and the most prominent point on the labial surface of the incisor (IB). 3- Upper lip distance to vertical plane was measured as the perpendicular distance between the most anterior point on the upper lip (Ls) and the vertical reference plane. 4- Lower lip distance to vertical plane was measured as the perpendicular distance between the most anterior point on the lower lip (Li) and the vertical reference plane. 5-Maxillary incisors to vertical plane was measured as the perpendicular distance between the most anterior point on the crown of the upper incisor and the vertical reference plane. 6-Mandibular incisors to vertical plane was measured as the perpendicular distance between the most anterior point on the crown of the lower incisor and the vertical reference plane.

\section{Statistical analysis:}

Data were saved on an Excel spreadsheet and then transferred to SPSS software package (SPSS for Windows 98, version 10.0, SPSS Inc, Chicago, Ill) for statistical analysis. The mean and standard deviations of the differences between pre- and posttreatment for each of the hard and soft tissue measurements were calculated. The effect of orthodontic correction was assessed using paired $t$-tests to determine the significance of change between pre- and posttreatment. Then data were then subjected to Pearson $r$ correlation analysis, which calculated the correlation coefficient between dental and soft tissue variables used in the study. 


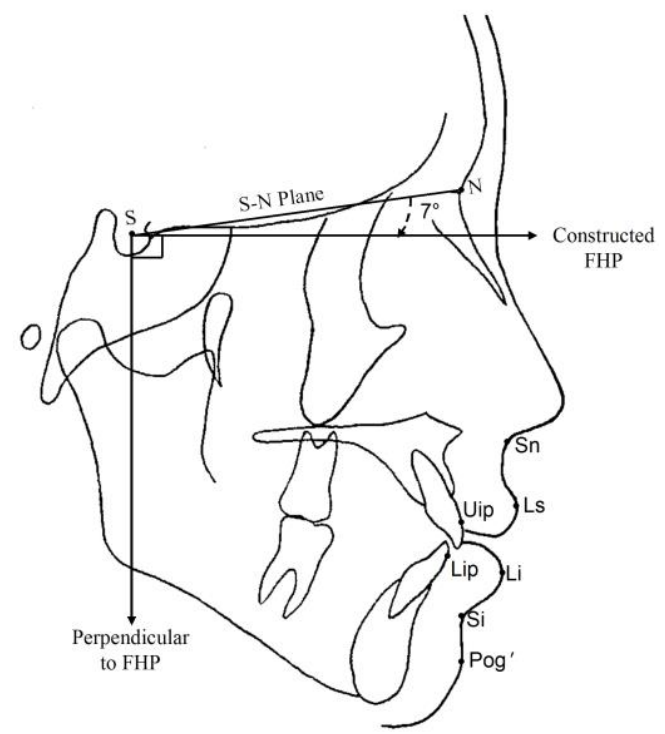

Fig.1. Soft tissue landmarks, hard tissue landmarks and reference planes

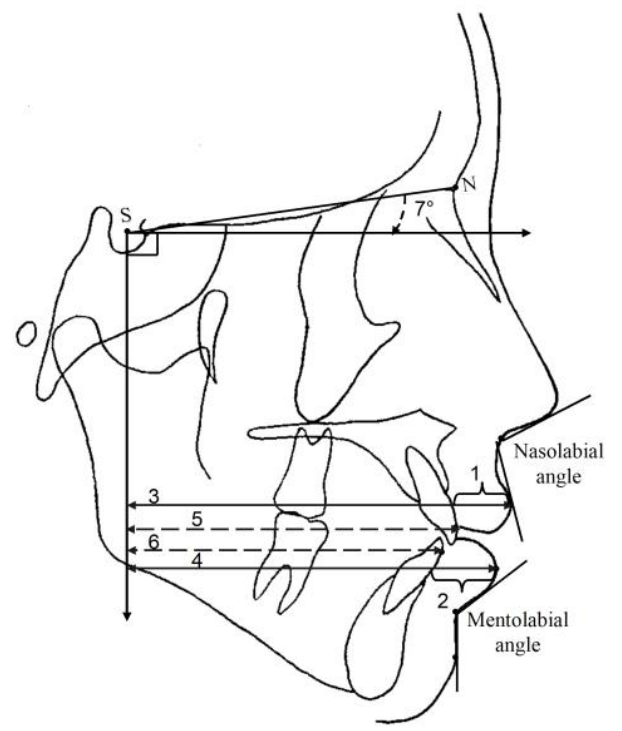

Fig.2. Angular and linear measurements used. 


\section{RESULTS}

Table 1 lists the mean of pre, post treatment and differences of mean of the variables studied. Statistically significant post treatment differences were observed for the : Nasolabial angle, Labiomental angle, Upper lip thickness, Lower lip thickness, Upper lip retraction, Lower lip retraction, Upper incisor retraction and Lower incisor retraction $(P<0.01)$.

Table 2 shows the Pearson correlation coefficients (r) of dental and soft tissue treatment changes.

Table (1): Mean values and standard deviations of pre, post and difference of angular and linear measurements and results of statistical comparisons.

\begin{tabular}{|l|c|c|c|c|c|c|c|}
\hline \multirow{2}{*}{\multicolumn{1}{|c}{ Variable }} & \multicolumn{2}{|c|}{ Pretreatment } & Post treatment & \multicolumn{2}{c|}{ Difference } & \multirow{2}{*}{$\boldsymbol{P}$} \\
\cline { 2 - 7 } & Mean & SD & Mean & SD & Mean & SD & \\
\hline Nasolabial angle & 96.85 & 8.85 & 106.2 & 9.66 & 9.35 & 2.80 & $0.000 * * *$. \\
Labiomental angle & 130.4 & 8.75 & 132.3 & 7.82 & 1.95 & 1.63 & $0.000 * * *$. \\
Upper lip thickness & 14.65 & 1.33 & 16.5 & 1.36 & 1.85 & 0.51 & $0.000 * * *$. \\
Lower lip thickness & 15.75 & 2.25 & 17.1 & 2.38 & 1.35 & 0.40 & $0.000 * * *$. \\
Upper lip retraction & 91.8 & 5.53 & 88 & 5.85 & -3.8 & 0.76 & $0.000 * * *$. \\
Lower lip retraction & 93.15 & 4.98 & 89.1 & 4.95 & -4.05 & 0.66 & $0.000 * * *$. \\
Upper incisor retraction & 85.15 & 2.18 & 80.4 & 2.06 & -4.75 & 0.65 & $0.000 * * *$. \\
Lower incisor retraction & 79.4 & 2.35 & 74.4 & 2.58 & -5.00 & 0.85 & $0.000 * * *$. \\
\hline
\end{tabular}

S.D. $=$ Standard deviation $* * * \mathrm{P} \leq 0.001$ (Highly significant)

Table 2 : Pearson correlation coefficients (r) of dental and soft tissue treatment changes.

\begin{tabular}{|l|l|l|}
\hline \multicolumn{1}{|c|}{ Variable 1 } & \multicolumn{1}{|c|}{ Variable 2 } & R value \\
\hline Nasolabial angle difference & Upper incisors retraction difference & $0.600^{* *}$ \\
Labiomental angle difference & Upper incisors retraction difference & 0.279 \\
Upper lip thickness difference & Upper incisors retraction difference & 0.039 \\
Lower lip thickness difference & Upper incisors retraction difference & -0.349 \\
Lower lip retraction difference & Upper incisors retraction difference & $0.749^{* *}$ \\
Lower lip retraction difference & Lower incisors retraction difference & $0.781^{* *}$ \\
Upper lip retraction difference & Upper incisors retraction difference & $0.884^{* *}$ \\
Upper lip retraction difference & Lower lip retraction difference & $0.843^{* *}$ \\
\hline
\end{tabular}


Egyptian

Orthodontic Journal

\section{DISCUSSION}

A well-balanced and harmonious soft-tissue facial profile is an important consideration in orthodontic diagnosis and treatment planning. Orthodontists have long recognized that the extraction of premolars often is accompanied by changes in the soft tissue profile. At times, these changes result in substantial improvements in the profile and frequently justify the extraction of teeth in patients without other indications. At other times, however, premolar extraction can lead to a flatter profile. ${ }^{25}$

Because of the negative perception of protrusive dentition and lips in most cultures, many patients with bimaxillary protrusion seek in most orthodontic care to decrease this procumbency.

The goals of orthodontic treatment of bimaxillary protrusion include the retraction and retroclination of maxillary and mandibular incisors with a resultant decrease in soft tissue procumbency and convexity. This is most commonly achieved by the extraction of four first premolars followed by the retraction of anterior teeth using maximum anchorage mechanics.

The aim of this study was to explore the effect of incisors retraction upon lip positions. With this in mind, subjects with Class I bimaxillary protrusion were selected to evaluate this effect. The entire sample was adults who passed the active period of growth to be sure that there is a negligible amount of facial growth remaining.

An accurate determination of the effects of treatment on the integumental profile requires removal of the effects of growth as well as gender differences. Few investigators have separated the effects of treatment from growth. Russell and Nelson ${ }^{26}$ and Park et al. ${ }^{27}$, reported that the relationship of soft-tissue reference points could not be shown to be directly affected by retraction of the incisors because of the masking effect of growth. In this study only female patients were used who past adolescence at the start of treatment.

Again to maintain uniformity in the sample average mandibular plane angle subjects were selected as orthodontic treatment may affect the profile differently in long-faced and short-faced individuals. ${ }^{28}$

This study showed that treatment with extraction of four premolars is very successful in reducing the dental and soft tissue profile procumbency seen in patients with bimaxillary protrusion, this finding is in consistent with the results obtained by Bills et al. ${ }^{29}$, who stated that "the extraction of four premolars can be extremely successful in reducing the dental and soft tissue procumbency seen in 
patients with bimaxillary protrusion, thus providing a stronger evidence-based rationale for this treatment modality." Also the results obtained is in agreement with $\operatorname{Tan}^{30}$ who studied orthodontic correction of bimaxillary protrusion in 50 Chinese adult patients and found favorable soft tissue and dental changes after the extraction of four premolars. Also support Lew ${ }^{31}$ when he investigated the profile changes after the extraction of four first premolars and orthodontic treatment of bimaxillary protrusion in 32 Asian adults. He reported significant improvement in upper and lower incisor protrusion, nasolabial angle, upper and lower lip length, and upper and lower lip protrusion.

As the profile varies according to malocclusion type, the present study used only Class I subjects having bimaxillary protrusion. On the other hand, skeletal variations may exist in subjects with a Class I molar relationship. This study compared the soft-tissue changes with corresponding incisors retraction by means of pretreatment and posttreatment lateral cephalometric radiographs.

\section{Nasolabial and Labiomental angles:}

The nasolabial angle is greatly affected by orthodontic treatment. All procedures should place this angle in the cosmetically desirable $102^{\circ} \pm 8^{\circ}$ range. ${ }^{32,33}$ An increase in the nasolabial angle may be due to a turned up nose or to lips that slant back. ${ }^{17} \mathrm{An}$ acute angle allows for maxillary incisor retraction; an obtuse angle suggests a maxillary retrusion with a need for protrusion of the maxillary incisors. ${ }^{34}$

The mean pre treatment nasolabial angle for the sample used in this study was $96.85^{\circ}$.This figure approaches that of Bills et al., ${ }^{29} 94^{\circ}$ but higher than that of $\operatorname{Tan}^{30}$, who determined the pretreatment nasolabial angle of Chinese patients with bimaxillary protrusion to be decreased at $86.68^{\circ}$. This could be attributed to the three different ethnic groups dealt with in the three studies in addition to this there may be some samples variations.

This result is another indication of the soft tissue procumbency seen in patients with this condition. The mean post treatment increase of the nasolabial angle was $9.35^{\circ} \pm 2.80^{\circ}$. This increase was highly significant, a finding which is in accord with that obtained by Caplan and Shivapuja ${ }^{35}$, Finnoy et al ${ }^{36}$, Hazar ${ }^{37}$ and Nelson ${ }^{38}$.

Drobocky and Smith ${ }^{25}$ examined the soft tissue profiles in 160 orthodontic patients treated by removing the 4 first premolars. The mean changes for the total sample included an increase of $5.2^{\circ}$ in the nasolabial angle and retraction of the upper and lower lips of 3.4 and $3.6 \mathrm{~mm}$ to the E-line, respectively. When 
they compared the profile changes to values representing normal (or ideal) facial esthetics, it was evident that extracting the 4 first premolars generally did not result in a "dished-in" profile. These findings are consistent with the results concluded in this study.

The labiomental angle plays an important role in the esthetic evaluation of the facial profile. In a study concerning soft tissue profile preference, De Smit and Dermaut ${ }^{39}$ reported that a flattening of the mental fold led to a more drastic loss of esthetic preference than a deepening. Nanda et al. ${ }^{40}$ determined that at 18 years the mean value of the angle $127.1^{\circ} \pm 12.9^{\circ}$ for the females.

The labiomental angle in this study showed a little but significant post treatment increase. This finding is consistent with the results of Caplan and Shivapuja ${ }^{35}$, Kocadereli ${ }^{41}$, Kusnoto and Kusnoto ${ }^{43}$,Drobrocky and Smith ${ }^{25}$ and Hazar $^{37}$ when they found a slight post treatment increase in the labiomental angle. But in a disagreement with the results obtained by Sheehan ${ }^{42}$ when he found a non significant post treatment decrease in the labiomental angle and concluded that the labiomental angle exhibited great variability in response to treatment in both the Caucasian and African American patients.

\section{Retraction of Lips:}

The ratio for the maxillary-incisor retraction to the upper-lip retraction has been a common computation in the past literature. For the upper lip, the ratio for the upper incisors retraction to upper lip retraction obtained in this study was 1.3:1.0(4.75 $\mathrm{mm}$ of upper incisor retraction to $3.8 \mathrm{~mm}$ of upper lip retraction). This result is approaching to those of Kusnoto ${ }^{43}$, Ramos et al. ${ }^{44}$ Tallas $^{24}$, Rains $^{45}$, Caplan ${ }^{35}$ and Kokodynski ${ }^{46}$ and in conflict with Rudee ${ }^{11}$, Roos ${ }^{47}$, Waldman $^{48}$, Bill' ${ }^{29}$ and Hershey ${ }^{14}$. The latter suggested that it may seem reasonable to accept that if the patient's initial incisor overjet was caused by extreme upper incisor proclination, then the subsequent correction (by retraction of the incisal edge with little backward displacement of the cervical point) tended to produce less lip change. On the other hand, more lip retraction was seen when the incisor retraction underwent translatory movement and the initial incisor inclination was close to normal.

This study showed that retraction of maxillary incisors caused the upper lip to retract significantly high. Analysis of correlation coefficients demonstrated a strong correlation $(\mathrm{r}=0.884)$ of the maxillary incisor and retraction of upper lip. This finding agrees with that of Rudee ${ }^{11}$ who found strong correlations for soft tissue and dental movements. Rains and Nanda ${ }^{45}$ found that the soft tissue response may vary for a given amount of tooth movement, they also found that 
the lower lip varied greatly with mandibular incisor movement, that the lower lip was more variable than the upper lip to differences in upper incisor movement, and that the upper lip was more variable with increased retraction of the maxillary incisor.

Other researchers stated that lip structure seems to have an influence on lip response to incisor retraction. Oliver ${ }^{18}$ found that patients with thin lips or a high lip strain displayed a significant correlation between incisor retraction and lip retraction, but patients with thick lips or low lip strain displayed no such correlation. In addition, Wisth ${ }^{19}$ found that lip response, as a proportion of incisor retraction, decreased as the amount of incisor retraction increased. This seems to indicate that the lips have some inherent support.

The lower lip, showed a significant posttreatment retraction.The mean retraction of the lower incisors of $5.0 \mathrm{~mm}$ was associated with an average lowerlip retraction of $4.05 \mathrm{~mm}$. these figures showed a high correlation which is consistent with the results obtained by Caplan and Shivapuja ${ }^{35}$ and Hazar et al, ${ }^{37}$

\section{Thickness of Lips:}

Changes in lip posture depend largely on the position of the incisors, therefore this study focused on how the retraction of the incisors affected lip thickness. Studies on lip changes with incisor movement have attempted to establish ratios that could serve as a guide for the clinician in treatment planning. According to Holdaway ${ }^{17}$ with a thick upper lip, it is not possible to protrude the upper lip by advancing the upper incisors. The thickness of the lips is a factor in the response to the orthodontic movement. When the upper lip thickness at the vermilion border is greater that $18 \mathrm{~mm}$, the upper lip usually changes very little when the upper incisor is retracted. When the upper lip is thinner than $12 \mathrm{~mm}$, the upper lip moves back as the teeth are retracted.

This study showed an increase in upper lip thickness with upper incisors retraction, the ratio of the change in upper lip thickness to upper incisor retraction was 1:2.57(1.85:4.75) which approaches the ratio obtained by Ricketts ${ }^{49}$ when he observed in a sample of 1000 treated patients with a "usual orthodontic problem" that the upper lip thickened on average $1 \mathrm{~mm}$ with $3 \mathrm{~mm}$ of maxillary incisor retraction.

Anderson et $\mathrm{al}^{50}$, found in their sample of 27 males and 43 females that the ratio for upper lip thickening to maxillary incisor retraction was $1: 1.5 \mathrm{~mm}$.

Talass et al, ${ }^{24}$ and Ricketts ${ }^{49}$ reported increases in upper lip thickness because of retraction of the maxillary incisor. In this study, there was an average 
increase in lip thickness of $1.85+0.51 \mathrm{~mm}$ during the course of treatment. Brock ${ }^{51}$ et al, reported only a slight increases in lip thickness in the inferior half of the upper lip and a small decrease in the superior half. Diels et $\mathrm{al}^{52}$ in a study of black females, reported similar increase in lip thickness at labrale superiorus with retraction of the maxillary incisors. Likewise, Oliver ${ }^{18}$ found that white females had little or no change in lip thickness, measured at superior labial sulcus, with maxillary incisor retraction.

On the contrary ,Hershey ${ }^{14}$ described $0.71 \mathrm{~mm}$ reduction with one $\mathrm{mm}$ of retraction of upper incisors retraction, similar results were obtained by Ramos et. $\mathrm{al}^{44}$ when he found a decrease in upper lip thickness of $0.7-0.8 \mathrm{~mm}$ during the course of treatment. Other reports have suggested lip retraction with incisor retraction with a wide range in correlation. ${ }^{11,14,24,45,47,48}$

The results of this study would suggest that extreme changes in the dental or skeletal factors, such as excessive retraction of the anterior teeth, may lead to adverse facial profile changes. It would seem possible for the clinician to carefully manage the decision of extraction and also either first or second premolar extraction while still protecting the lateral profile.

\section{CONCLUSIONS}

- Extraction of four premolars greatly reduces soft tissue procumbency seen in patients with bimaxillary protrusion.

- The Nasolabial angle, Lower lip retraction and Upper lip retraction correlates well with upper incisors retraction.

- Lower lip retraction correlates well with lower incisors retraction.

- Upper lip retraction correlates well with the lower lip retraction.

- Labiomental angle does not show good correlation with upper incisors retraction.

- Lower lip thickness does not correlate with upper incisors retraction.

- Upper lip thickness showed poor correlation with Upper incisors retraction.

\section{REFERENCES}

1. Angle EH. Malocclusion of the teeth. 7th ed. Philadelphia: S.S. White Dental, 1907.

2. Broadbent HB. The face of the normal child. Angle Orthod 1937;7:183-208. 
3. Brodie AG. On the growth of the human head from the third month to the eighth year of life. Am J Anat 1941;68: 209-16.

4. Tweed $\mathrm{CH}$. The Frankfurt mandibular plane angle in orthodontic diagnosis. Classification, treatment planning and prognosis. Am J Orthod Oral Surg 1946;32:175-204.

5. Björk A. The face in profile. Lund: Berlingska Boktryckeriet, 1947.

6. Riedel RA. Esthetics and its relation to orthodontic therapy. Angle Orthod 1950;20:168-78.

7. Burstone CJ. The integumental profile. Angle Orthod 1959; 29:93-104.

8. 8-Downs WB. Analysis of the dentofacial profile. Angle Orthod 1956;26:191-212.

9. Bloom, L.: Perioral profile changes in orthodontic treatment, Am J Orthod 47: 371-379, 1961

10. Riedel, R. A.: An analysis of dentofacial relationships, Am J Orthod. 43: 103-119, 1957

11. Rudee, D. A.: Proportional profile changes concurrent with orthodontic therapy, Am J Orthod. 50: 421-434, 1964

12. Stoner, M. M., and Lindquist, J. T.: A cephalometric evaluation of 57 cases treated by Dr. C. H. Tweed, Angle Orthod. 26: 68-98, 1956

13. Angelle, P. L.: A cephalometric study of the soft tissue changes during and after orthodontic treatment, Trans. Eur. Orthod. Soc., pp. 267-280, 1973

14. Hershey, H. G.: Incisor tooth retraction and subsequent profile change in post adolescent female patients. Am J Orthod. 61: 45-54, 1972

15. Neger, M. A.: A quantitative method for the evaluation of the soft-tissue facial profile, Am J Orthod. 45: 738-751, 1959

16. Subtelny, J. D.: The soft tissue profile, growth and treatment changes, Angle Orthod. 31: 105-122, 1961

17. Holdaway RA. A soft tissue cephalometric analysis and its use in orthodontic treatment planning. Part I. Am J Orthod Dentofac Orthop. 1983; 84:1-28.

18. Oliver BM. The influence of lip thickness and strain on upper lip response to incisor retraction. Am J Orthod Dentofac Orthop.1982;82:141-8 
Egyptian

Orthodontic Journal

19. Wisth PJ. Soft tissue response to upper incisor retraction in boys. Br J Orthod 1974;1:199-204

20. Perkins RA. Change in lip vermilion height during orthodontic treatment. [Thesis.] lowa City: University of lowa, 1987

Quoted from: Bishara, Cummins, Jakobsen, and Zaher, Dentofacial and soft tissue changes in Class II Division 1 cases. Am J Orthod Dentofac Orthop 1995 Jan (28-37)

21. Burstone, C. J.: Lip posture and its significance in treatment planning, Am J Orthod. 53: 262-332, 1967

22. Ricketts, R. M.: Foundation for cephalometric communication, Am J Orthod.46: 330, 1960

23. Cohen J, Cohen P. Applied multiple regress/correlation analysis for the behavioral sciences. Hillsdale, New Jersey: Erlbaum, 1983.

Quoted from: McFarlane, Frydman, McCabe, and Mamandras : Nasal tip deflection with LeFort I . Am J Orthod Dentofac Orthop 1995 Mar (259-267)

24. Talass MF, Talass L, Baker RC. Soft tissue profile changes resulting from retraction of maxillary incisors. Am J Orthod Dentofac Orthop 1987;91:385-94

25. Drobocky OB, Smith RJ. Changes in facial profile during orthodontic treatment with extraction of four first premolars. Am J Orthod Dentofac Orthop1989;95:220-30.

26. Russell DM and Nelson RT. Facial soft tissue profile change in the North American black with four first premolar extractions. Md State Dental Assoc 1986;29(1):24-8

27. Park S,Kudlick EM,Abrahamian A.Vertical dimension changes of the lops in the North American black patients after four-first premolar extraction. Am J Orthod Dentofacial Orthop 1989;95:220-30

28. Lai J, Ghosh J, Nanda R, Effects of orthodontic therapy on the facial profile in long and short vertical facial patterns Am J Orthod Dentofacial Orthop 2000;118:505-1

29. Bills D., Handelman C, BeGole E. Bimaxillary Dentoalveolar Protrusion: Traits and Orthodontic Correction. Angle Orthod 2005;75:333-339

30. Tan TJ. Profile changes following orthodontic correction of bimaxillary protrusion with a preadjusted edgewise appliance. Int $\mathbf{J}$ Adult Orthod Orthognath Surg 1996;11:239-51. 
31. Lew K. Profile changes following orthodontic treatment of bimaxillary protrusion in adults with Begg appliance. Eur J Orthod 1989;11:375-81.

32. Legan HL, Burstone CJ. Soft tissue cephalometric analysis for orthognathic surgery. J Oral Surg 1980;38:744-51.

33. Epker BN, Fish LC. Dentofacial deformities, vol I. St Louis:C.V. Mosby; 1986.

34. Arnett GW, Bergman RT. Facial keys to orthodontic diagnosis and treatment planning: part I. Am J Orthod Dentofac Orthop 1993;103:299-312.

35. Caplan MJ, Shivapuja PK. The effect of premolar extractions on the soft tissue profile in adult African American females. Angle Orthod 1997;129-36.

36. Finnoy JP, Wisth PJ, Boe OE. Changes in soft-tissue profile during and after orthodontic treatment. Eur J Orthod 1987;9:68-78.

37. Hazar S, Akyalcin S and Boyacioglu H. Soft Tissue Profile Changes in Anatolian Turkish Girls and Boys Following Orthodontic Treatment With and Without Extractions. Turk J Med Sci 2004;34:171-178.

38. Nelson RI III. Facial soft-tissue profile changes in the North American Black with four first bicuspid extractions. Thesis, Howard University College of Dentistry, Dept. of Orthodontics, Washington, DC:1984.

Quoted from: Sheehan S. Effects of Premolar Extraction on the Facial Profile of African Americans and Caucasians: A Comparative Study. OC-J Volume II Issue 1 April 1997

39. De Smit A, Dermaut L. Soft tissue profile preference. Am J Orthod 1984; 86:67-73.

40. Nanda RS, Meng H, Kapila S, Goorhuis J. Growth changes in the soft tissue facial profile. Angle Orthodontist 60: 177-190, 1990.

41. Kocadereli I.Changes in soft tissue profile after orthodontic treatment with and without extractions. Am J Orthod Dentofacial Orthop 2002;122:67-72

42. Sheehan S. Effects of Premolar Extraction on the Facial Profile of African Americans and Caucasians: A Comparative Study. OC-J Volume II Issue 1 April 1997

43. Kusnoto $\mathrm{J}$ and Kusnoto $\mathrm{H}$. The effect of anterior tooth retraction on lip position of orthodontically treated adult Indonesians. Am J Orthod Dentofacial Orthop 2001;120:304 -7 
44. Ramos A L , Sakima M T, Pinto A S, Bowman S J. Upper Lip Changes Correlated to Maxillary Incisor Retraction-A Metallic Implant Study. Angle Orthod 2005;75:499-505.

45. Rains MD, Nanda R. Soft tissue changes associated with maxillary incisor retraction. Am J Orthod 1982;81:481-8.

46. Kokodynski RA, Marshall SD, Ayer W, Weintraub NH, Hoffman DL. Profile changes associated with maxillary incisor retraction in the postadolescent orthodontic patient. Int J Adult Orthod Orthognath Surg 1997;12:129-34.

47. Roos N. Soft-tissue profile changes in Class II treatment. Am J Orthod 1977;72:165-74.

48. Waldman BH. Change in lip contour with maxillary incisor retraction. Angle Orthod 1982;52:129-34.

49. Ricketts RM. Cephalometric synthesis. Am J Orthod 1960; 46:647-73

50. Anderson JP, Joondeph DR, Turpin DL. A cephalometric study of profile changes in orthodontically treated cases ten years out of retention Angle Orthod 1973;43:324-35.

51. Brock RA,Taylor RW,Buschang PH and Behrents RG. Ethnic differences in upper lip response to incisor retraction. Am J Orthod Dentofacial Orthop 2005;127:683-91

52. Diels RM, Kalra V, DeLoach N, Powers M, Nelson SS. Changes in soft tissue profile of African-Americans following extraction treatment. Angle Orthod 1995; 65:285-92. 